\title{
Jona in der Jesustradition des ersten Jahrhunderts auf der Grundlage literarischer und archäologischer Zeugnisse
}

Der neue Fund eines Jona-Fisch-Graffitos aus dem Jerusalem vor der Katastrophe des Jahres $70 \mathrm{n}$. Chr. gibt Anlass, die Geschichte des Jonamotivs in der Jesusüberlieferung erneut aufzurollen und die Trägergruppen der verschiedenen Jona-Zeugnisse zueinander in Beziehung zu setzen.

\section{Das Jonamotiv in der literarischen Tradition}

\subsection{Ebene des historischen Jesus}

1. Q 11,31f: ${ }^{1}$ Im von nachösterlicher Christologiebildung noch unberührten Doppelwort von Südkönigin und Ninivitern vergleicht sich Jesus mit dem Gericht und Umkehr predigenden Propheten Jona, dessen Gerichtsbotschaft das Unheil von Ninive abwendete, weil die Stadt Ninive die in der Gerichtspredigt gegebene Chance be- und ergriff.

In doppelter Weise verleiht Jesus in diesem sprachlich kunstvollen ${ }^{2}$ Logion seiner Umkehrpredigt an Israel Nachdruck. Das Jesu Hörer Herausfordernde liegt darin, dass ausgerechnet Heiden ihre Chance ergriffen, sich Gott zuzuwenden (dgl. Q 7,9; 10,13f). Das heißt, Jesu Hörerschaft in Israel wird bei ihrer Ehre gepackt, Heiden nicht nachzustehen. ${ }^{3}$ Zusätzlich spornt

1 Vorausgesetzt wird im Folgenden der griechische Text der Critical Edition of $Q$ des International Q Projects, leicht zugänglich z.B. bei HofFMANN/HeIL, Spruchquelle.

2 Wie in der Rhetorik üblich, werden Paradigmen aus der Geschichte aneinandergereiht (vgl. Hebr 11): Salomo-Südkönigin, Jona-Ninive (Q 11,31f); ähnlich Noah-Sintflut und Lot-Sodom in Q 17,26-30. Vgl. z.B. DORMEYER, Literaturgeschichte, 143ff. Der Text scheint aus dem Aramäischen übersetzt; das artikellose „Königin vom Süden“ in Q 11,31 semitisiert.

3 Ähnlich Röm 11,13f. Jesus reizt darüber hinaus auch in Q 17,26-30 mit dem Blick auf Nichtisraeliten seine Hörer sich umzuorientieren. In diesem Text werden allerdings nicht positive Beispiele paganen Hinwendens zu Gott genannt, sondern negative, die Sintflutgeneration und die Sodomiter, die Jesu Hörerschaft in Israel zur Warnung dienen sollen: Israel wird gleichfalls im nahen Endgericht aus der Gottesherrschaft ausgegrenzt werden, wenn es sich nicht auf die in Jesus anbrechende (Q 11,20) Gottesherrschaft einlässt (Q 12,8). Kohärenz 
das Logion dadurch an, dass es behauptet, Jesu Botschaft überbiete die des Jona (Q 11,32); sein weisheitlich und prophetisch geprägtes Verkünden sei „mehr als Salomo“ und „mehr als Jona“. Das Mehr liegt im Kontext jesuanischer Verkündigung darin, dass Jesus endgültiges Richten und endgültiges Herrschen Gottes ansagt.

Wie sich das Schicksal der Niniviter an ihrer Reaktion auf die Jonapredigt entschied, besiegelt der Hörer Haltung zu Jesu Umkehrbotschaft deren Schicksal im Gericht: Kehren sie nicht um, werden die Nichtjuden aus dem Süden und aus Ninive, die sich zu Gott kehrten und gerettet wurden, sie im Zeugenstand des Endgerichts „verurteilen“. Auch im Menschensohnwort Q 12,8f formuliert Jesus die Korrespondenz zwischen jetziger Reaktion auf sein Wirken und künftigem Gerichtsgeschick, ohne sich dort selbst mit dem Weltrichter zu identifizieren. Am vorösterlichen Ursprung von Q 11,31f zu zweifeln, bietet sich kein Anlass. Jesus zeigt in seinem Rückgriff auf Jona das Selbstverständnis eines Propheten.

Das Motiv des Auferstehens verbindet Q 11,31f bezeichnenderweise noch nicht mit Jona, sondern mit der Südkönigin und den Ninivitern, die Gott $\mathrm{zu}$ Endgericht und Zeugenstand wiedererwecken wird. Eine Auferstehungssymbolik der Seemonstergeschichte Jonas dagegen bleibt außerhalb des jesuanischen Horizonts.

Noch der Erste Clemensbrief teilt bei seiner Jonarezeption den jesuanischen Fokus auf den Umkehr predigenden Propheten: „Der Herr gab von Geschlecht zu Geschlecht denen, die sich zu ihm bekehren wollten, Gelegenheit zur Buße ... Jonas kündigte den Ninivitern Katastrophe an, doch die, welche umkehrten ..., erlangten Rettung" (1Clem 7,5ff). Auch der christliche Rahmen vom Vierten Esrabuch behielt denselben Fokus bei: Als Bußprediger für die paganen Niniviter wird Jona zu einem der prophetischen Führer des neuen Volkes aus den Heiden, also der Heidenkirche stilisiert (4Esr 1,39f). Auf die Seemonstergeschichte und ihre mögliche Auferstehungssymbolik wird kein Bezug genommen.

\section{Q 11,16.29f:}

(16) Einige aber ersuchten vom ihm ein Zeichen. (29a) Er aber sagte: Diese Generation ist eine schlechte Generation. (b) Sie fordert ein Zeichen, (c) und ein Zeichen wird ihr nicht gegeben werden, (d) nur das Zeichen des Jona. (30) Denn wie Jona den Ninivitern

zwischen diesen Logien wird sichtbar, nicht nur Kontinuität zur Gerichtspredigt des Täufers (Q 3,7ff.16b-17). Dass Q 11,31f Jesu Verkündigung entgegenstehen könnte, weil Jesus „bis zum Schluss seines Wirkens Israel zur Buße gerufen“ habe (Luz, Matthäus, 275), ist nicht einsichtig: Das Drohwort soll gerade Israel zur Neuorientierung antreiben. Auch Luz (275) verwirft deshalb schlussendlich den Gedanken und versteht Q 11,31f wie Q 13,28 und vielleicht sogar Q 10,13f ,als letzten, dringlichen Appell an Israel“. 
zum Zeichen wurde, so wird auch der Menschensohn dieser Generation zum Zeichen werden.

Wer nicht Q 11,16.29f von vornherein als Gemeindebildung verstehen möchte, wozu kein hinreichender Grund besteht, sondern von einem vorösterlichen Kern ausgeht, hat nicht weniger als sieben mögliche Modelle für den Überlieferungsprozess des Logions V. 29f abzuwägen. Ein vorösterlicher Ursprungstext umfasste:

\section{Alternative [I] V. 29a-c. ${ }^{4}$}

[I.1.-2.] Nachösterlich wird zunächst die Ausnahme V. 29d angefügt, später V. 30, um diese Ausnahme zu erklären. - Variante zu letzterem: Möglicherweise existiert der V. 30 als selbständiges, aber nachösterlich gebildetes Logion bereits, bevor er an V. 29d angefügt wird. ${ }^{5}$

[I.3.-4.] Nach Ostern werden V. 29d.30 zusammen angefügt. - Variante: Möglicherweise existiert V. 30 bereits vorher als selbständiges Logion, und V. 29d wird als Brücke zwischen beide Logien konstruiert. ${ }^{6}$

\section{Alternative [II] V. 29a-d.}

[II.1.-2.] V. 30 wird nachösterlich angefügt. ${ }^{7}$ - Variante wiederum: Möglicherweise existiert V. 30 bereits vorher als selbständiges Logion.

Alternative [III] V. 29f. ${ }^{8}$

Wie nähmen sich Modelle I und II auf der Ebene des historischen Jesus aus? Die Passage Q 11,16.29a-c(d?) (par Mk 8,11f) stellt eine selbständige Tradition dar, die erst sekundär aufgrund gemeinsamer Stichworte („Jona“, „dieses Geschlecht“, „Niniviter") mit dem Drohwort Q 11,31f verbunden wurde; ${ }^{9}$ sie behandelt ein anderes Thema als Q 11,31f, indem sie als Scheltrede das Zeichenfordern der Jesuskritiker und Jesu Reaktion darauf wieder-

4 Dafür, den ältesten Bestand (ohne Jona) in Q 11,29c enden zu lassen, plädierte z.B. LÜHRMANN, Logienquelle, 42.

5 Letzteres erwägt z.B. Luz, Matthäus, 274. Die Frage, wann V. 30 als selbständiges Logion entstanden sein könnte, ist m.E. eindeutig zu beantworten: nach Ostern. Vorher würde der Vers nur in Verbindung mit einer Zeichenforderung wie V. 16 Sinn machen, so dass es aberwitzig wäre, V. $30 \mathrm{zu}$ einem von den V. 16.29 unabhängigen vorösterlichen Logion zu deklarieren. Nach Ostern hingegen könnte V. 30 als selbständiges Logion gebildet worden sein (s.u. Textabschnitt [c] nach Anm. 25).

6 Letzteres (Modell I.4.) erwägt SATo, Prophetie, 283.

7 Für Q 11,30 als sekundäre Erweiterung z.B. KLOPPEnBorg, Formation, 130; SchüRMANN, Reich, 164.

8 Für ursprüngliche Einheit von Q 11,29f als Jesuswort z.B. BAYER, Predictions, 131.

9 Gegen ursprüngliche Einheit spricht, dass sich nur Q 11,32 mit Q 11,29f thematisch verbindet (d.h. Salomo im jetzigen Zusammenhang überschießt) und Markus zwar eine 
gibt. Da Wundertaten ambivalent sind - auch ein böser Geist vermag, Dämonen auszutreiben (Q 11,15) - fordern die Kritiker ein eindeutiges Zeichen vom Himmel zum Ausweis der göttlichen Vollmacht Jesu. Jesus verweigert sich dem Ansinnen (Modell I). Am vorösterlichen Anker der Tradition zu zweifeln, bestünde kein Anlass.

Die Frage stellt sich lediglich, ob auch bereits Q 11,29d - „außer dem Zeichen des Jona“ - dem ältesten vorösterlichen Traditionsbestand angehörte (Modell II). Markus hätte den Jonahinweis dann als zu rätselhaft herausgestrichen ${ }^{10}$ denn er kannte nicht das authentische Jesuslogion Q 11,31f, das seinem Verständnis auf die Sprünge hätte helfen können (s. gleich).

Das Modell II wäre denkbar. Für den historischen Jesus hätte das „Jonawunder“ in seinem eigenen Wirken in Israel darin bestanden, dass seine Predigt bei vielen in Israel, bei zumindest einem halben Tausend (1Kor 15,5), ja, zuweilen wohl sogar bei Heiden (Q 7,9; vgl. 10,13f), Erfolg zeigte - wie es bei Jonas Predigt gewesen war (Q 11,32) -, denn diese vielen kehrten um, folgten ihm nach und wandten sich dem anbrechenden Gottesreich zu (,Jonawunder“ in Modell II = Predigterfolg Jesu). Ähnlich verbuchte Paulus, nach Legitimation für seinen Apostolat befragt, seine erfolgreiche Gemeindegründung in Korinth als Beweis seines Gottgesandtseins, so dass ihm andere Legitimationserweise überflüssig erschienen (2Kor 3,1-3). Entgegen dem üblichen Sprachgebrauch, der in der Regel kosmische Ereignisse als „Zeichen“ gelten ließ (s.u. Anm. 18), ist ein derart verstandenes Jona-Zeichen unscheinbar, gleichsam ein „Nichtzeichen“, das den „Begriff ,Zeichen" verfremdet", ${ }^{11}$ darin aber genau der jesuanischen Zeichenverweigerung entspricht.

In dem prophetischen Scheltwort von Q 11,29a-d hätte Jesus sich - wie in Q 11,32 - dem Propheten Jona wiederum typologisch entsprechend gesehen. Q 11,29a-d, für sich allein genommen, ergäbe mithin durchaus einen kohärenten Sinn. ${ }^{12}$

Parallele zu Q 11,29a-c bietet, aber Q 11,31f nicht kennt. So auch Luz, Matthäus, 273 Anm. 8; 275.

10 Ähnlich z.B. KlopPenBorg, Formation, 130.

11 Luz, Matthäus, 279.

12 Nicht gesehen von z.B. Luz, Matthäus, 274, der V. 29a-d, für sich allein genommen, als unverständlich abtut. - In eine andere als hier vorgeschlagene Richtung weist ScнмiтT, Zeichen, 128, zur Interpretation des Jonazeichens. In den Vitae Prophetarum des 1. Jh. n. Chr. $(10,10 \mathrm{f})$ wird unter dem Eindruck des Jüdischen Krieges formuliert, dass Jona vorausgeahnt habe, dass das Ende nahe bevorstehen und Jerusalem zerstört werden würde, wenn ein Stein schreien (vgl. Hab 2,11) und viele Heiden in der heiligen Stadt sich aufhalten würden. Galten ein schreiender Stein und vermehrte Heidenpräsenz in Jerusalem als Jonazeichen? Die Prophetenviten würden das einzige Indiz (nicht einmal einen Beleg) für eine solche Vorstellung liefern. Und stammte Q 11,29d erst aus der Zeit des Jüdischen Krieges oder danach? Vor allem aber, wieso könnte ein schreiender Stein Jesu Vollmacht erweisen? Nicht Jesus (vgl. Lk 19,4044) hatte einen schreienden Stein als Omen des Jerusalemer Untergangs geweissagt, sondern 
Modell III. Falls auch noch Q 11,30 zur ursprünglichen Textebene gehörte, verschöbe sich der Sinn. Auf der Ebene des historischen Jesus könnte Q 11,29f paraphrasiert lauten:

(29) Es wird kein Zeichen zum Erweis des Gottgesandtseins Jesu geben ,außer dem des Jona. (30) Wie Jona den Ninivitern zum Zeichen“" wurde, ,so wird (Futur) der Menschensohn“ ein Zeichen zum Erweis der göttlichen Vollmacht Jesu „für diese Generation werden“, denn er wird diejenigen, die sich in Israel aufgrund der Jesuspredigt im Hier und Jetzt dem ankommenden Gottkönig zuwenden, im Endgericht retten und die anderen verurteilen.

Der Menschensohn wird somit bestätigen, dass die jetzige Reaktion der Hörer auf Jesu Verkündigen über deren eschatologisches Heil entscheidet, und so den vollmächtigen Charakter der Jesuspredigt im Nachhinein belegen - was die Aufgabe des geforderten Zeichens ist. Der Halbvers Q 11,30b läge auf derselben Argumentationsebene wie Q 12,8f. Im Rahmen der Verkündigung des historischen Jesus wäre er vorstellbar.

Noch von einer anderen Seite her gälte es, letzteres zu testen. Passt das „Menschensohn“ von Q 11,30b zur übrigen Verkündigung des historischen Jesus? Da in der synoptischen Tradition (a) „Menschensohn“ allein im Munde Jesu zu hören ist und dies obendrein häufig, (b) Jesus keine weitere eschatologische Hoheitsbezeichnung in den Mund nimmt, (c) nirgends offen sich mit dem Menschensohn identifiziert sowie (d) das frühe Entstehen einer nachösterlichen Menschensohnchristologie sich am besten erklärt, wenn irgendein vorösterlicher Anlass dafür gegeben war, liegt es nahe, einige Menschensohnworte dem historischen Jesus selbst zuzuschreiben, ${ }^{13}$ und zwar am ehesten die, welche ,Menschensohn“ nicht als Selbstbezeichnung verwenden, sondern vom Menschensohn im apokalyptischen Sinne ${ }^{14}$ als eschatologischem Weltenrichter und Retter in der dritten, das heißt, einer anderen Person als Jesus reden (v.a. in Q 17,26-30 und Q 12,8f/Mk 8,38). ${ }^{15}$ Q 11,30b würde diese Kriterien erfüllen und somit den Kohärenztest bestehen.

Zusammengefasst würde der vorösterliche Jesus nach Modell III auf die Forderung nach legitimierenden Zeichen hin antworten, dass es nur ein Zeichen geben werde: Der noch nicht mit Jesus identifizierte Menschensohn wird durch sein eschatologisches Gerichtshandeln nachträglich beweisen, dass Jesu jetziges Wirken göttlich bevollmächtigt ist - und zwar dadurch, dass er als Weltenrichter die Rezipientenreaktion gegenüber Jesu jetzigem Wirken zum Maßstab seines Gerichtshandelns erheben wird.

den Prophetenviten zufolge Jona. Das heißt, Q 11,26d in der Schmittschen Lesart wäre sinnlos im Kontext der Zeichenforderung der Jesuskritiker.

13 So z.B. auch BECKer, Jesus, 251f.

14 Zur Figur des individuellen Weltenrichters, die auf dem noch kollektiv verstandenen „Menschensohn“ von Dan 7,13.22.27 basiert, s. evtl. Targum zu Dan 7,13f (dazu KocH, Menschensohn, 82f), dann vor allem 1Hen 37-71 (spätestens 1. Jh. n. Chr.) und nach $70 \mathrm{n}$. Chr. 4Esr und syrBar.

15 Würde der historische Jesus in diesen Texten von sich selbst sprechen, müssten wir ihm eine „Verhüllungsstrategie unterstellen“ (BECKER, Jesus, 252), worauf nichts weist. 
Das Futurum in Q 11,30b spricht dagegen, diesen Halbvers auf die Linie der Menschensohnworte einzutragen, die auf ein gegenwärtiges Wirken des Menschensohns abheben (Q 9,58; 7,34; Mk 2,10.28 u.ö.). ${ }^{16}$

Freilich, ein Problem steht dem Modell III im Wege. Was bedeuteten bei dieser Lesart die Formulierungen „Zeichen des Jona“ und „Jona wurde für die Niniviter zu einem Zeichen“" (29d-30a)?

(a) War Jonas Auftritt als Gerichtsprophet für Ninive ein warnendes Vorzeichen vor dem Gottesgericht? ${ }^{17}$ Eine solche Lesart würde aus dem Rahmen des üblichen Sprachgebrauchs herausfallen, in dem kosmische Geschehen als warnende Vorzeichen galten. ${ }^{18}$ Zwar wäre diese Schwierigkeit überwindbar, wenn der ungewöhnliche Semeion-Gebrauch als Ausdruck der jesuanischen Zeichenverweigerung begriffen würde (s.o.). Doch lohnt es nicht, diese Spur weiter zu verfolgen, denn es bliebe einerseits im Dunkeln, wieso eine warnende Gerichtsandrohung im Kontext von V. 29 einen legitimierenden Charakter haben könnte, und andererseits, worin die Analogie zwischen dem Gericht predigenden Jona und dem im Eschaton Jesus legitimierenden Menschensohn eigentlich bestünde. ${ }^{19}$

(b) Eher wäre im Rahmen des Modells III vorauszusetzen, dass die Niniviter von der wundersamen Errettung Jonas aus dem Meer wussten ${ }^{20}$ und durch dieses Wunderzeichen sich überzeugen ließen, Jonas Buß- und Gerichtspredigt ernst zu nehmen. Das Jonawunder von Jon 2 hätte die göttliche Legitimation des Gerichtspropheten (in Jon 3) ausgewiesen - so wie das wundersame Erscheinen des eschatologischen Menschensohnrichters die göttliche Legitimation Jesu erweisen wird. Möglich wäre diese Lesart. Sie beinhaltete noch nicht ein auferstehungstypologisches Deuten des Jonawunders von Jon 2. Doch steht zweierlei gegen diese Lesart. Einerseits wäre die Vorstellung, dass die Niniviter vom Meerwunder erfuhren und dieses als legitimierendes Zeichen begriffen, im Judentum singulär. ${ }^{21}$ Andererseits wird wiederum die Analogie Jona/Menschensohn nicht recht plausibel. Das tertium comparationis wäre lediglich ein schwaches, denn es bestünde darin, dass in beiden Fällen eine Predigt zwar göttlich legitimiert würde, jedoch nur durch höchst unterschiedliche Ereignisse. Beide Wider-

16 Gegen z.B. Luz, Matthäus, 275, wo das Futur ignoriert wird.

17 Vgl. z.B. KLopPenBorg, Formation, $132 \mathrm{f}$.

18 Z.B. Lk 21,7.11.25; Mt 24,3; Mk 13,4.24f; Joel 3,3; Jes 13,10; 34,4.

19 Beide unterscheiden sich deutlich. Jona wirkte im Gegensatz zum Menschensohn nicht als Richter, sondern nur als ansagender Prophet. Vor allem führte seine Gerichtspredigt zur Rettung Ninives, während der Menschensohn „diese böse Generation“ (Q 11,29) verurteilen wird.

20 Vgl. z.B. BAYer, Predictions, 138.

21 Von Jonas Errettung aus dem Meer hören zwar Jonas Hausgenossen (3Makk 6,8) und die Seeleute (PRE 10), jedoch nicht die Niniviter. Auch wenn PRE 10fin das Erretten Jonas aus dem Meer ausdrücklich den ,Zeichen“ zuordnet, ist Ninive nicht Adressat. 
haken, vor allem der zweite, sind der Wahrscheinlichkeit dieser Lesart abträglich, so dass Modell III den Modellen I und II unterliegt.

\subsection{Q 11,16.29f auf nachösterlicher Verstehensebene}

Auch wenn das Futurum ein Deuten auf gegenwärtiges Leben, Wirken oder (Buß)-Predigen des Menschensohns ausschließt, ${ }^{22}$ bleiben zum Test verschiedene Lesarten, denen gemeinsam ist, dass sie nunmehr vom InEinssetzen des Menschensohns mit Jesus ausgehen. ${ }^{23}$

(a) Wie Jona als Bußprediger den Ninivitern zum Zeichen wurde (11,30a), so werde ich als Weltenrichter-Menschensohn dieser bösen Generation zum Zeichen werden (11,30b). Freilich, worin die Jona/Menschensohn-Analogie läge, bliebe auch bei dieser Lesart undeutlich, da jener nur Gericht ansagte, welches dann nicht eintrat, während dieser richten und „diese böse Generation" verurteilen wird. Ungeklärt bliebe zudem, inwiefern ein Bußprediger zum (legitimierenden) „Zeichen“ werden kann.

(b) Gangbarer wäre: Wie Jonas wundersame Errettung aus dem Meer (Jon 2) den Ninivitern zum Zeichen wurde, welches den Propheten legitimierte, ${ }^{24}$ so wird mein wundersames Auftreten als himmlischer Weltenrichter-Menschensohn die göttliche Legitimation meines jetziges Wirkens nachträglich erweisen. Ich werde dann diesem Geschlecht zeigen, dass seine Reaktion auf meine jetzige Predigt über sein eschatologisches Heil entscheiden wird, und so belegen, dass meine jetzige Verkündigung vollmächtig ist. ${ }^{25}$ Doch worin liegt wiederum die Analogie zwischen Jona und Jesus? Lediglich darin, dass beider Wirken als göttlich bevollmächtigt legitimiert

22 S.o. bei Anm. 16. Die Lösungsfindung durch Spekulation über eine aramäische Textversion mit präsentischem Sinn aufzublähen, verbietet sich methodologisch.

23 Für den Sinn macht es deshalb keinen Unterschied, ob „Menschensohn“ lediglich als Umschreibung der ersten Person verstanden wird oder nicht. Wer das Futurum ernst nimmt, wird so oder so an der eschatologischen Richterfunktion nicht vorbei kommen - wenn er nicht von vornherein auf die Auferstehungsinterpretation (s. gleich Punkt c) zugehen will. Zu philologisch-aramaistischen Problemen der Erste-Person-Umschreibung VöGTLE, „Gretchenfrage“, $31-64$.

24 Nach Ostern hätten Christen so die für das Judentum einmalige Vorstellung geschaffen, dass die Niniviter um die Jonaerrettung von Jon 2 wussten (so wie später in PRE 10 die Seeleute vom Fischwunder wussten; s.o.). Methodisch stehen wir vor dem Dilemma, dass wir auf irgendeiner Traditionsebene das naive Voraussetzen der Kenntnis des Fischwunders bei den Ninivitern ansiedeln müssen - wenn wir nicht bei der soeben zurückgewiesenen Lesart (a) stehenbleiben wollen.

25 MORA, Signe, 57ff, möchte mit Blick auf Q 11,31f für die Q-Ebene noch weiter konkretisieren: Der auferstandene Menschensohn-Weltenrichter wird dann die bekehrten Heiden ,diese böse Generation“ anklagen lassen. Die Analogie Jonazeichen/Menschensohnzeichen wird so jedoch noch mehr vernebelt. 
werden kann. Die Legitimationsarten selbst haben nichts miteinander gemein.

(c) Wesentlich glatter fällt die Jona/Jesus-Analogie aus, wenn bereits auf der nachösterlichen Q-Ebene das Jonawunder von Jon 2 auferstehungstypologisch als dem Auferstehen Jesu analog gedeutet wurde: Kein Zeichen, „,nur das des Jona. Wie Jona für die Niniviter ein Zeichen wurde“, indem er aus dem Bauch des Seeungeheuers nach drei Tagen von Gott befreit wurde (so dass den Ninivitern seine Legitimation als gottgesandter Gerichts- und Bußprediger deutlich wurde), so wird Jesus, der Menschensohn, nach drei Tagen aus dem Schlund des Todes befreit und auferweckt werden als ein göttliches Zeichen, welches „für dieses Geschlecht" das Wirken des irdischen Jesus nachträglich legitimieren wird. Der Vers Q 11,30 läge dann auf der Linie der nachösterlichen Worte vom sterbenden und auferstehenden Menschensohn (Mk 8,31; 9,9.31; 10,33f.45; 14,21.41), auch wenn in Q diese Linie sonst nicht weiter verfolgt wird. Matthäus freilich hätte den Sinn von Q 11,30 „richtig“ verstanden, als er in Mt 12,40 expressis verbis Jon 2 typologisch auf Jesu Sterben und Auferstehen deutete.

\subsection{Zusammenfassung zu Q 11,16.29f mit Blick auf Mt 12,40; 1Kor} 15,4 und zeitgenössische jüdische Quellen

Unter dem Strich ist der Überlieferungsprozess von Q 11,16.29f nicht bis ins Letzte zu klären. Auch wenn Modell III die geringste Wahrscheinlichkeit für sich verbuchen kann, verbleiben die Modelle I und II als gleichwertige Möglichkeiten für den, der nicht voreilig und grundlos den gesamten Text zur nachösterlichen Bildung erklären will. Mindestens V. 29a-c wird im vorösterlich-jesuanischen Kontext verständlich; doch gilt letzteres auch für V. 29a-d. Vers 30 dagegen reiht sich erst nach Ostern an.

Mit Jesu Totenauferstehen wurde das Jonazeichen erst auf der matthäischen Textebene expressis verbis in eins gesetzt. Doch auch für Q 11,16.29f ist dieses Verständnis gut möglich; ja, es erwies sich auf der nachösterlichen Verstehensebene m.E. als das plausibelste (s.o. Punkt c). 


\begin{tabular}{|c|c|c|}
\hline Vers & Historischer Jesus & Nachösterlich \\
\hline $29 \mathrm{~d}$ ohne 30 & $\begin{array}{l}\text { Erfolg der jesuanischen Predigt als } \\
\text { Legitimationserweis } \\
\Rightarrow \text { nicht nur Modell I, auch Modell II ergäbe } \\
\text { Sinn }\end{array}$ & $\begin{array}{l}\text { Sinn wie } \\
\Leftarrow \text { bzw. } \Downarrow\end{array}$ \\
\hline $29 \mathrm{~d}-30$ & $\begin{array}{l}\text { 30b: das Jesus legitimierende „Jonazeichen“ } \\
=\text { der Menschensohn wird (Futur) die jetzige } \\
\text { Hörerreaktion auf Jesu Predigt als Kriterium } \\
\text { im Endgericht verwenden (Jesus nicht mit } \\
\text { Menschensohn identisch) } \\
\text { 29d-30a: entweder } \\
\text { (a) Jonazeichen für Niniviter = Jonas Buß- } \\
\text { predigt = warnendes Vorzeichen vor dem } \\
\text { Gottesgericht } \\
\Rightarrow \text { Dilemma I,* } \\
\Rightarrow \text { Dilemma II,** } \\
\text { oder } \\
\text { (b) das Jona in Ninive legitimierende Zeichen } \\
=\text { seine Rettung aus dem Meer } \\
\Rightarrow \text { Dilemma II,** } \\
\Rightarrow \text { Modell III ergibt keinen rechten Sinn }\end{array}$ & $\begin{array}{l}\text { dito, wie mittlere Spalte } \\
\text { (jedoch Jesus = Menschen- } \\
\text { sohn) } \\
\text { dito, entweder } \\
\text { (a) dito } \\
\Rightarrow \text { Dilemma I, } \\
\Rightarrow \text { Dilemma II, } \\
\text { oder } \\
\text { (b) dito } \\
\Rightarrow \text { Dilemma II } \\
\Rightarrow \text { Lösungen (a) und (b) } \\
\text { überzeugen nicht, deshalb: }\end{array}$ \\
\hline $\begin{array}{l}30 \text { (allein } \\
\text { oder mit 29d) }\end{array}$ & & $\begin{array}{l}\text { (c) Die Rettung aus dem } \\
\text { Fischbauch war den Ninivitern } \\
\text { ein die Predigt des Jona } \\
\text { legitimierendes Zeichen, wie } \\
\text { die Rettung des Menschensohn } \\
\text { Jesus aus dem Tod ein dessen } \\
\text { Predigt legitimierendes } \\
\text { Zeichen für diese Generation } \\
\text { sein wird. }\end{array}$ \\
\hline $\begin{array}{l}\text { Dilen } \\
\text { legiti } \\
\Rightarrow \mathrm{ke} \\
\text { Dilen } \\
\Rightarrow \mathrm{ke}\end{array}$ & \multicolumn{2}{|c|}{$\begin{array}{l}\text { Dilemma I: eine warnende Gerichtsandrohung kann im Kontext von V. } 29 \text { keinen Jona } \\
\text { legitimierenden Charakter haben; sie selbst bedarf der Legitimation } \\
\Rightarrow \text { kein Sinn } \\
\text { Dilemma II: Analogie Jona/Menschensohn nicht plausibel } \\
\Rightarrow \text { kein Sinn }\end{array}$} \\
\hline
\end{tabular}

Tab. 1: Sinnfüllungen für „Jonazeichen“. 
Über eine Generation vor Matthäus hatte bereits in den dreißiger oder vierziger Jahren die Formel von 1Kor 15 Jesu Verweilen im Tod in auffälliger Parallelität zu Jon 2 auf drei Tage beziffert. ${ }^{26}$ Die Zeitangabe bedeutet, dass dem Petrus entweder am ,dritten Tag“ nach der Kreuzigung tatsächlich seine erste Vision widerfuhr oder dass die erste Vision nach der Kreuzigung nachträglich in Ermangelung genauer Zeitangaben in Anlehnung an Jon 2 und andere Schriftstellen auf den dritten Tag datiert wurde. ${ }^{27}$ Wie immer dem sei, in jedem Fall sahen die Autoren der Formel zwischen Jesu Tod-Auferstehungs-Schicksal und Jon 2 (sowie Hos 6,2; Gen 22,4-19) eine Parallele, denn sie versahen „am dritten Tag“ mit dem Zusatz „nach den Schriften". 1Kor 15,4 stellt somit den ersten literarischen Hinweis auf eine auferstehungstypologische Deutung von Jon 2 dar. Der Jonatext selbst hatte den Anstoß dazu gegeben, denn bereits Jon 2,3f.6 verglich des Propheten Bedrängnis mit dem Tod.

Einmal ins christliche Denken eingeführt, erfreute sich Jonas typologisch deutbare Seeungeheuergeschichte von Jon 1,1-2,11 besonderer Beliebtheit im Neuen Testament: Zwölf der 19 möglichen Jonabuchanklänge im Neuen Testament beziehen sich auf diese Geschichte. ${ }^{28}$

Im zeitgenössischen Judentum dagegen wurde die Seemonstergeschichte von Jon 2 sehr lange nicht auferstehungstypologisch gelesen, wenn sie denn überhaupt interessierte. Tob 14,4.8 rekurriert allein auf Jonas Unheilsprophetie über Ninive. Für alexandrinische Juden des 1. Jh. v. Chr. (3Makk 6,8f) wird Jonas Erretten aus dem Fischbauch zwar zur Trostgeschichte; von paganen Nachbarn bedrängt, wenden sie sich mit Blick auf Gottes Erretten

26 Weniger nah dagegen andere alttestamentliche Parallelen. Hos 6,2 redet formelhaft von zwei bzw. drei Tagen. In Gen 22,4-19 finden das Opfer bzw. die schlussendliche Schonung Isaaks vorm Tode am dritten Tage statt. In Ex 19,11.16 wird Gott am dritten Tage aktiv, aber es geht dort nicht um Erretten aus Todesnot, sondern um den Sinai-Bundesschluss. Nach Philo, VitMos I 163, beträgt die Distanz des Exodus von Ägypten nach Kanaan drei Tagesreisen; nach Josephus, Ant II 315, die Reise Israels von Ägypten ans Rote Meer ebenfalls drei Tage. In Gen 42,17f befreit nicht Gott, sondern Josef am dritten Tag seine Brüder aus der Haft (noch entfernter die Parallelen Esr 8,15; Est 4,16-5,3 sowie die anderen von BerR, 56 zu 22,4f, und EstR, 9 zu 5,1ff, genannten alttestamentlichen Stellen).

27 Darüber hinaus könnte die jüdische Sitte, am „dritten Tage“ nach einer Bestattung zum Grab zurückzukehren, um zu prüfen, ob der Beigesetzte wirklich tot war, einen weiteren Grund für die Auferstehungsdatierung geliefert haben. $\mathrm{Zu}$ dieser Sitte s. eine Baraita im allerdings erst aus dem 3. Jh. n. Chr. stammenden jüdischen Traktat Semahot (8,1). „Drei““ ist statt „dreißig“ in der Überzahl der Manuskripte zu lesen; nur so macht das Prüfen von Lebenszeichen am Verstorbenen Sinn (vgl. SAFraI, Home, 784f). Wurde darüber hinaus der „dritte Tag“ unter dem Eindruck von Logien wie Lk 13,31ff formuliert? So LeHMAnN, Auferweckt, 185.

281 Kor 15,4; Mt 12,40; 16,4 (von Matthäus im Sinne von 12,40 verstanden); 8,24; 23,35; Mk 4,37; 4,41 (bis); Lk 21,25; Joh 11,50; Apg 27,19; Apk 11,13fin. Die drei kursiven Stellen heben auf die Auferstehungsthematik ab. Kein Bezug auf die Seeungeheuergeschichte, aber auf das Jonabuch: Mt 2,10; 11,21; 12,41; 26,38; Mk 14,34; Lk 10,13; 11,32. 
des Jona aus dem Meer, des Daniel aus der Löwengrube und der Freunde aus dem Feuerofen bittend an Gott. Doch knüpfen sie keine Auferstehungshoffnungen an Jonas Seeabenteuer-Geschichte. ${ }^{29}$

Phrygische Juden augusteischer Zeit listen Jona neben den Erzvätern und anderen Propheten zwar als einen der im Eschaton von den Toten Auferweckten auf, doch wird die Fischgeschichte damit in keinen Zusammenhang gebracht (Sib. II 248, zugehörig zur jüdischen Grundschrift). In den Vitae Prophetarum geben palästinische Juden des 1. Jh. n. Chr. in 10,6 die Legende wieder, Jona sei der Sohn der Witwe von Zarepath gewesen, ${ }^{30}$ den Elija von den Toten auferweckte, um, ihm zu zeigen, dass es unmöglich ist, vor Gott wegzulaufen“. ${ }^{11}$ Das Auferweckungsmotiv knüpft sich hier gerade nicht an die nur kurz an anderer Stelle $(10,2)$ erwähnte Seeabenteuergeschichte an, sondern an die Elija-Zarepath-Geschichte von 1Kön 17. Josephus erzählt zwar die Seegeschichte, interessiert sich aber nicht für mögliche Auferstehungssymbolik (Ant IX 206-214). ${ }^{32}$

Erst spätere jüdische Quellen nähern sich - vermutlich unter christlichem Einfluss - einer solchen Typologie an. PRE 10 vergleicht den Fischbauch wie Jon 2,3 mit der Tiefe der Scheol: ${ }^{33}$

Er zeigte ihm den Gehinnom, ... die unterste Scheol ... Herr der Welt, ... hebe mich empor! Du wirst genannt der, welcher tötet und lebendig macht: Siehe, meine Seele ist dem Tode nahe, so mache mich lebendig!“

MekhJ Ex 12,1 (2a) schließlich bringt, angelehnt an Jon 1,12-15, wie die Christen das Motiv der Stellvertretung ins Spiel:

Jona ging weg, um sich selbst im Meer dem Untergang zu weihen ... Ebensolches findest du bei den Vätern und Propheten, dass sie sich selbst für Israel dahingaben.

29 Ähnlich ist in den sechzehn „Hellenistischen Synagogengebeten“ des 2./3. Jh. n. Chr. (erhalten in den Büchern 7 und 8 der Apostolischen Konstitutionen) der Jona im Walfischbauch $(6,11)$ einer der Prototypen des zu Gott betenden Gerechten $(11,3)$. Aber auch hier liegt kein Bezug zur Auferstehungsthematik vor. Ebenso wenig wie in der Mishna (mTaan 2,4): „Der, der Jona im Fischbauch antwortete, wird dir antworten und dein Schreien heute hören. Gesegnet seist du, Herr, der du in Notzeit auf Beten antwortest.“

30 Wesentlich später ebenso PRE 33; MTeh Ps 26,7 (110b).

31 Auch PRE 10 und bSan 89ab werden sich später für Jonas Auftragsverweigerung interessieren; bSan 89ab obendrein (wie Modell II oben) für die Umkehr der von Jonas Gerichtsprophetie Bedrohten.

32 Dasselbe gilt für die auch Jona einschließende Liste mehrerer Propheten, die sich eschatologisch geäußert hätten: AscJes 4,22 (ein freilich christlicher Zusatz vom Ende des 1. Jh. n. Chr.).

33 „Bauch der Scheol“ auch im Midrash Jalq Jon 4. MTeh Ps 26,7 (110b) dagegen: „Er wurde im Schlund der Fische geläutert ... aber er starb nicht, sondern Gott gebot dem Fisch, Jona aufs trockene Land zu speien." 


\section{Der neue Jona-Fisch-Graffito in einem Jerusalemer Felsengrab}

\subsection{Der Fund in seinem Kontext}

Pünktlich zur Passionszeit 2012, kaum dass der Aschermittwoch verstrichen war, wartete publikumswirksam der Dokumentarfilmer Simcha Jacobovici mit einer neuen archäologischen Überraschung zu den frühesten Jesusanhängern auf. Fünf Jahre zuvor hatte er zusammen mit Oscarpreisträger James Cameron (Titanic, Avatar) Schlagzeilen mit der kühnen These gemacht, ein Felsengrab im Jerusalemer Wohnviertel Talpiyot berge die Knochenkisten der Familie Jesu. Die Theorie ging wie die Titanic unter. ${ }^{34}$

Doch dieses Mal arbeitete das Dokumentarfilmer-Team seriöser, mit Lizenz der israelischen Antikenbehörde und am Arm professioneller Stützen..$^{35}$ Fast drei Kilometer südöstlich der Jerusalemer Altstadt, wieder im Wohnviertel Talpiyot, unweit eines Einkaufszentrums und kaum 45 Meter vom umstrittenen „Jesus"-Familiengrab entfernt, führte das Team vom Keller eines Apartmentkomplexes aus eine Roboterkamera endoskopisch in ein unterirdisches Mausoleum ein. Der israelischen Antikenverwaltung war die Felsengruft seit Bauarbeiten des Jahres 1981 bekannt. ${ }^{36}$ Versiegelt und überbaut, wurde sie nie ausgegraben - auch jetzt nicht, um ultraorthodoxen Nachbarn entgegenzukommen, die die Totenruhe hüten.

Die Grabstätte datiert ins 1 Jh. n. Chr. Vor der Katastrophe von 70 n. Chr., als noch in Ossuarien zweitbestattet wurde, setzte hier draußen vor der antiken Stadt in ländlicher Umgebung, in der Nähe von Zisternen, einer Olivenpresse und eines jüdischen Ritualbads eine Gruppe über ein Dutzend Tote bei, darunter auch Kinder. Von drei Seiten der quadratischen Zentralkammer $(3,5$ x 3,5m) gehen neun bis zu über zwei Meter lange spitzgiebelige Grabschächte ab, die säuberlich ausgehauen und wie der Haupteingang mit einem Steinblock verschlossen wurden. Eine typisch jüdische Gruft Jerusalems.

Nach der Verwesung betteten die Angehörigen die Gebeine in acht Kalksteinossuarien um. Doch mindestens sieben Individuen liegen noch ausgestreckt in den Schächten. Entweder stand ihnen dieser bescheidene Luxus nicht $\mathrm{zu}$, oder es kam nicht mehr zum Umbetten, weil die Gruppe das Mausoleum nach der Eroberung Jerusalems im Jahre 70 n. Chr. aufgab.

34 S. z.B. LAmPE, MEXPI, 355-366. Der Artikel zeigt, dass auch die angeblich so schlagenden statistischen Argumente des Jacobovici-Teams nicht ziehen.

35 TABOR, Report; DERS., Hebrew.

36 Vgl. z.B. Kloner, Survey, 84; DERs./Zissu, Necropolis, 342, mit Plan. 
Professionelle jüdische Steinmetze dekorierten sieben der Ossuarien mit ornamentalen Bändern und Rosetten, die dem Gebot der Tora genügten, Bilder von Lebewesen zu vermeiden (Dtn 5,8f).

Während in der Zeit des Ersten Tempels das Gebot, keine Lebewesen darzustellen, vielfach übertreten wurde, zeichnete sich in der Phase des Zweiten Tempels, insbesondere seit den Hasmonäern, die ihre Münzen ohne Herrscherkopfbilder prägten, ein bilderloser Trend ab, der bis zum Bar Kochba-Aufstand währte. ${ }^{37}$ Fast ausschließlich pflanzliche, geometrische und architektonische Motive schmückten Sarkophage und Ossuarien. Aber auch die striktere Zeit ließ Ausnahmen zu - etwa Herrscherköpfe auf Münzen einiger Nachfolger Herodes des Großen, Tierbilder in Antipas' Palast in Tiberias, im Westen Jerusalems im vermutlich sadduzäischen Jason-Grab die Darstellung eines Kaufmanns neben drei Schiffen, auf Fresken Jerusalemer Privathäuser Vogeldarstellungen und über dem Hauptportal selbst des herodianischen Tempels einen goldenen Adler. ${ }^{38}$ Darstellungen von Lebewesen können im 1 . Jh. n. Chr. also nicht zwingend für christliche Heterodoxie sprechen.

Solche Kistchen ließen sich in Werkstätten kaufen; individualisieren mussten die Käufer sie selbst. Auf zwei Knochenkisten finden sich in griechischer Schrift Namen geritzt (MAPA, ${ }^{39}$ was freilich auch ,Herrin“ bedeuten könnte, und ION...). Auf einer dritten wurde zwischen zwei professionell vorgefertigte Dekorbänder eine vierzeilige griechische Kritzelei gedrängt. Sind Epitaphe in Jerusalemer Gräbern an sich bereits selten - sie versuchten meist, Verletzungen der Grabesruhe abzuwehren ${ }^{40}$-, so findet der Inhalt des Graffitos in keiner der rund 900 zeitgenössischen Grabstätten in und um Jerusalem eine Parallele:

$$
\begin{aligned}
& \Delta \mathrm{IO} \Sigma \\
& \text { IAIO } \\
& \Upsilon \Psi \Omega[\Sigma \mathrm{EI}] \\
& \mathrm{A}^{4} \mathrm{~B}^{41}
\end{aligned}
$$

37 S. mit Beispielen u.a. Foerster, Architecture, $971 \mathrm{f}$.

38 S. EBD. 976.985, ferner 984.999.1001f, auch unten nach Anm. 65, sowie einen Ochsenkopf auf einem diasporajüdischen Ossuar in Kammer B der Grabhöhle 2 auf dem Gelände des Aceldama-Konvents des Kidrontals: bei Avni/Greenhut/Ilan, Three New Burial Caves, $209 f(A b b$.).

39 Vgl. dgl. CIIP Nr. 97.200.262.477.517.563, drei davon griechisch; auch Ruth 1,20.

40 CIIP Nr. 359.375.385.451.460.466.528.602.604.605, auch 287.

41 So die wahrscheinlichste lectio der Z. 4 (neben auch möglichen АГІ $\Omega$, АПО [NEKP $\Omega N$ ?], AПB); s. die Photos in TABOR, Report, 40. Bei einer vokativischen Bitte („Himmlischer Gott, erhöhe!“”, wie Tabor liest) wäre eher $\Delta \mathrm{IE}$ zu erwarten, nicht $\Delta \mathrm{IO} \Sigma$ (vgl. LSJ, s.v. $\triangle \mathrm{IE})$. Es sollte deshalb nicht imperativisch $\mathrm{Y} \Psi \Omega[\Sigma \mathrm{ON}]$ ergänzt werden. Aber auch die 1. Person, ,ich erhöhe/preise“, legt sich angesichts eines Nominativs in Z. If nicht nahe. Sie müsste um den hohen Preis erkauft werden, dass $\triangle \mathrm{IO} \Sigma$ als Genitiv eines ZEY $\Sigma$ verstanden und Z.1f, auf die 1. Person bezogen, als ,Zeus IAIO zugehörig“ übersetzt würde, wie BAUCKHAM, Patio Tomb, vorschlägt: „Belonging to Zeus IAIO, I, Hagab, exalt (you/him).“ Warum ein Mann jüdischen Namens so etwas auf die Knochenkiste einer Gruft jüdischer Machart gekritzelt haben sollte, wäre zu klären. Obendrein assoziiert selbst die Umwelt (Diodorus u.a.) Iao eindeutig mit dem Judengott (s.u. Anm. 43). Eine Zeus-Iaio-Assoziation müsste für die Zeit vor 70 n. Chr. erst 
„Der Himmlische/Gott/wird erhöhen/Agb.“ Letzteres kürzt am ehesten den seltenen Namen Agabos ab; ein gleichnamiger Christ wirkte zur selben Zeit, im zweiten Drittel des 1. Jh., in Jerusalem als Prophet (Apg 11; 21). Ergänzen wir $\mathrm{A} \Gamma[\mathrm{A}] \mathrm{B}[\mathrm{O} \Sigma]$, war ein Agabos der Autor des prophetischfuturischen Satzes - was die Identifikation mit dem Agabos der Acta herausfordert, ohne bewiesen werden zu können. Lesen wir $\mathrm{A} \Gamma[\mathrm{A}] \mathrm{B}[\mathrm{ON}]$, war dieser der Verstorbene selbst. Eine weitere Lösung wäre, $А Г В$ als griechische Umschrift eines hebräischen „Heb empor!“ zu verstehen, die den Erhöhungsgedanken der vorangegangenen griechischen Zeile in bilingualer Weise wiederholte. ${ }^{42}$ Doch mutierte dann der indikativische griechische Aussagesatz im Hebräischen wenig passend zu einem Imperativ.

„Erhöhen“ drückte in zahlreichen frühchristlichen Texten den Auferstehungsgedanken aus (Apg 2,33; 5,31; Joh 12,32.34; 3,14; 8,28; Phil 2,9; vgl. LXX Ps 9,14; 29,2). All dies spricht zwar noch nicht zwingend für einen judenchristlichen Charakter des Ossuariums. Der Name Agabos ist im Ursprung jüdisch (Hagab: Esr 2,46; Neh 7,48), und Pharisäer hofften ebenfalls auf ein Auferstehen, ohne dies freilich, soweit bislang erkennbar, in Gräbern kundzutun. Aufmerken lässt jedoch die Form des Gottesnamens. Sie stellt die griechische Umschrift des hebräischen Tetragramms dar. ${ }^{43}$ Fromme Juden schrieben das Tetragramm zwar beim Kopieren ihrer Bibel, jedoch niemals in Gräbern, die sie als kultisch unrein empfanden (Num 19,16). Auf fast 700 jüdischen Inschriften Jerusalems findet sich nichts Vergleichbares. ${ }^{44}$

Als weiteren Tabubruch, in diesem Fall des Bilderverbots, bildet die Schmalseite eines anderen Ossuars ein Lebewesen $a b:{ }^{45}$ einen geschuppten

nachgewiesen werden. Auf einem magischen Täfelchen ist noch die jüdische Assoziation (IAIO $\Omega$ neben Adonai und Sabaoth; s.u. Anm. 43) deutlich erkennbar.

42 Hagbeh, Hiphil Imperativ von gbh. So Tabors Lösung (TABOR, Report, 19) mit Verweis auf Ez 21,31 und 4Q 431f2,8. Das letzte Wort über den Graffito ist noch nicht gesprochen, wie lebhafte Online-Dispute (mit z.T. abwegigen Vorschlägen!) auf http://asorblog.org zeigen.

$43 \mathrm{Vgl}$. IA $\Omega$ bereits in vorchristlicher Zeit bei Diodorus, bibl. I 94,2: ,unter den Juden ... der Gott, der als Iao angerufen wird“", fußend auf Poseidonius, frg. 134,16 (ed. Theiler); dann Irenäus, haer. I 1,7; I 14,2; Origenes, Joh. II 1,7; Origenes, Cels. VI 31,21; VI 32,19.23; Eusebius, d.e. IV 17,23; X 8,28; Epiphanius, pan. I 287,2; I 410,9; I 437,18; II 36,22; Johannes Chrysostomus, hom. in ps. LV 653,62; schließlich Hymni Anonymi (Hymni e papyris magicis collecti; pagan, röm. Kaiserzeit, ed. Heitsch) frg. 5,14f (der Schreiber ruft den Engel des Zeus, den göttlichen Jahwe und R[aphael], der Himmel und Erde beherrscht, an: TON $\triangle \mathrm{IO} \Sigma$ АГГЕ $\Lambda$ ON $\Theta E[I] O N$ IA $\Omega$ etc.); Hippiatrica Parisina 1026,2.8 (IA $\Omega$ und IAH) (9. Jh. n. Chr.); u.v.m. Andere Belege bei TABOR, Report, 15 Anm. 32. Wegen des zweiten Iotas besonders wichtig ist IAIO $\Omega$ auf einem Täfelchen, das offensichtlich Engel, nämlich Adonai, Sabaoth, Iaioo (= Jahwe) und Bel, beschwört, Schwäche und Tod zu schicken (bei JoRDAN, Notes, $117-$ 119; vgl. PGM III 41ff).

44 Überblick über 692 bekannte Inschriften in CIIP.

45 Das Bilderverbot bricht auf einem dritten Ossuar auch die primitive Strichzeichnung (Fig. 17 bei TABOR, Report, 39) eines Tieres mit vier Beinen, Schwanz und Kopf - wenn es sich bei der Zeichnung nicht wieder um ein in diesem Fall stilisiertes Tetragramm handelt. 
Fisch, der in die Tiefe abtaucht; nur der hintere Teil mit Schwanzflosse ist zu sehen. In der linken Hälfte der Frontseite wird dasselbe Motiv wiederholt, diesmal mit dem gesamten Fisch und Jona im Maul: Aus dem leicht geöffneten Fischmaul schaut ein kugelförmiger Gegenstand heraus, durchzogen von zahlreichen Ritzlinien (Abb. 1) - als wollte der „Künstler“ das Seegras andeuten, das sich nach Jon 2,6 um den Kopf des Jona schlang. ${ }^{46}$

Eine ernstzunehmende Alternative, die mit der Deutung auf Jon 2 konkurrieren könnte, ist m.E. trotz lebhafter Blog-Debatten auf asorblog.org nicht in Sicht. Der geschuppte Fisch auf der Ossuarfront ist zum Beispiel nicht als funerales Monument (Nephesch) interpretierbar, ${ }^{47}$ ein solches stünde bei unserem Ossuar verunglückt auf dem Kopf. Gegen eine NepheschDeutung oder auch eine Vasen-Interpretation sprechen darüber hinaus die geschuppte Schwanzflosse auf der Ossuarschmalseite sowie sechs weitere kleine Fische am oberen Ossuarrand, die das Motiv der Frontseite maritim umrahmen. ${ }^{48}$

Mit dem neuen Fund aus der Zeit vor $70 \mathrm{n}$. Chr. liegt bei der jetzigen Sachlage der weitaus früheste ikonographische Beleg für das Jona-FischMotiv und seine auferstehungstypologische Deutung in funeralem Kontext vor.

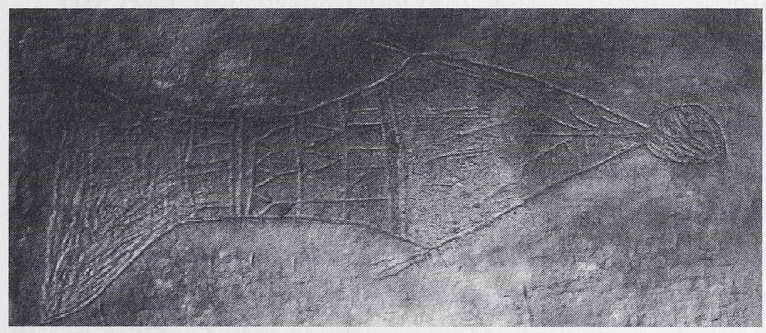

Abb. 1: Linke Frontseite des Jona-Fisch-Ossuars (um $90^{\circ}$ linksgedreht; Abdruck mit freundlicher Erlaubnis von James D. Tabor, University of North Carolina/Charlotte, USA).

46 Auf Seegras deutet auch TABOR, Report, 21. Nach TABOR, Hebrew, sind darüber hinaus im Kopf des Fisches die hebräischen Buchstaben des Namens „Jonah“, ausgehend vom Auge des Fisches, erkennbar, was freilich nicht jeden überzeugen wird.

47 Nephesh-Darstellungen in CJO Nr. 183.213.231.378.399.815. Keine könnte mit einem Fisch verwechselt werden.

48 An der anderen Schmalseite ist zudem eine Art „Fenster“ mit Fensterkreuz zu sehen - was vielleicht auf die „Riegel der Erde“ von Jon 2,7 anspielen könnte (so TABOR, Report, 22), die sich hinter Jona in Todesnot schlossen. Das vom Tode Dahingerafft-Werden drückt sich möglicherweise auch darin aus, dass der Fisch auf der linken Frontseite mit Jona nach unten schwimmt. TABOR, Report, 21, interpretiert etwas anders: Der Fisch schwimmt auf den Boden $\mathrm{zu}$, auf dem das Ossuar steht, also aufs Land zu, um Jona ins Leben zurückzuspeien. In der rechten Fronthälfte, die gegenwärtig von einem anderen Ossuar zugestellt ist, so dass nur Teile sichtbar werden (drei Photos in http://jamestabor.com), steht in einem rechteckigen Rahmen ein zweites Rechteck, das sich in mehrere horizontale Bänder gliedert. In jedem Band wogt eine Wellenlinie auf und ab, so dass an eine (weitere?) Darstellung der „Riegel der Erde“ gedacht werden kann. 


\subsection{Judenchristliche Provenienz}

Wie der literarische Befund zeigte, interessierten sich nichtchristliche Juden erst wesentlich später als Christen für das Jona-Auferstehungs-Motiv. Es waren mithin zunächst nur frühe nachösterliche griechischsprachige Jesusanhänger, für die Jona zum Symbol der Totenauferweckung wurde (1Kor 15,4; Mt 12,40 in Aufnahme griechischen Q-Textes; wahrscheinlich auch Q 11,29f selbst, s.o.). Ab dem dritten Jahrhundert schmückten wiederum Christen ihre Katakomben mit diesem tröstenden Motiv, ${ }^{49}$ während jüdische archäologische Belege bislang fehlen..$^{50}$ All dies spricht dafür, dass auch das neue Jona-Fisch-Ossuar, das das Bilderverbot ignorierte und - parallellos für zeitgenössische jüdische Gräber - Auferstehungshoffnung ausdrückte, von Christen benutzt wurde, die sich noch vor Matthäus an auferstehungstypologischer Deutung von Jon 2 erfreuten.

Für Christlichkeit auch des zweiten, einen Vierzeiler bietenden Ossuars spricht nicht nur, dass es ebenfalls von Auferstehungszuversicht kündet, sondern auch, dass - wiederum ohne jüdische Parallele - der (transkribierte) hebräische Gottesname auf einen kultisch unreinen Grabgegenstand graviert wurde. Zudem hob vor allem im frühchristlichen Sprachgebrauch „erhöhen“ auf Auferstehung ab (s.o.).

Dass die gesamte Gruft von Christen benutzt wurde, ist damit freilich nicht ausgemacht, auch wenn im Jona-Fisch-Ossuar gleich mehrere Individuen zur Ruhe gebettet wurden und diese christliche Gebeinekiste an prominenter Stelle in der Gruft aufgestellt war: vorne in der ersten Nische, gleich rechts für die zum Eingang Hereinkommenden. ${ }^{51}$ Ebenso gut möglich ist, dass eine aus Juden und Judenchristen gemischte Gruppe die Gruft belegte.

Die jüdische Provenienz der gesamten Gruppe, also auch der Christen in ihr, zeigt sich am Typ der Grabanlage, an der in ihr gepflegten Bestattungsart, an der genannten Ossuar-Ornamentik vom Meißel professioneller jüdischer Steinmetzen, die das Bilderverbot achteten und von denen die Gruppe die Ossuarien erworben hatte, sowie daran, dass einige Gruppenmitglieder griechisch-hebräische Bilingualität (zumindest in IAIO) an den Tag legten.

49 Vgl. z.B. Nestori, Repertorio, 199; SNyder, Ante, 87.

50 Vgl. z.B. Goodenough, Symbols, $225 \mathrm{ff}$. Freilich, sein Postulat, die Beliebtheit des Motivs in christlichen Kreisen setze einen nichtchristlichen jüdischen Prototyp voraus, erweist sich im Lichte des neuen Fundes als voreilig.

51 TABOR, Report, 27. 
2.3 Nähe zu 1Kor 15,4, zum Jerusalemer Stephanuskreis und zu den nachösterlichen Tradenten der beiden Jona-Logien

Für Neutestamentler besonders brisant wird der Fund dadurch, dass sich Querverbindungen zu allbekannten Größen ziehen lassen.

(a) Mit den Schöpfern der Ossuarkritzeleien scheinen wir in dieselben hellenistisch-judenchristlichen Kreise vorzudringen, die in den dreißiger oder vierziger Jahren des 1 . Jh. in Jerusalem ${ }^{52}$ die Glaubensformel von 1 Kor 15 mit ihrer Jona-Auferstehungs-Typologie $(15,4)$ prägten: ,,auferweckt am dritten Tage nach den Schriften“, das hieß vor allem, nach Jon 2 und Hos 6,2 (Gen 22,4-19). Schriftverweise und Formulierungsbausteine wie „Kephas“ und ,die Zwölf" sprechen für judenchristliche Autoren der Glaubensformel. ${ }^{53}$ Sie sprachen Griechisch. ${ }^{54}$

Wie die Formelautoren gehörten auch die Mausoleumsbenutzer zum hellenistischen Judenchristentum, wie die auf den Ossuarien griechisch geschriebenen Namen und der im Wesentlichen griechische Ossuar-Vierzeiler zeigen. Es verlockt, beide auf diese Weise ähnlichen Personenkreise wenigstens teilweise zur Deckung zu bringen, da beide sich auch für auferstehungstypologische Deutung von Jon 2 interessierten.

(b) Aus der hellenistischen Judenchristenschaft Jerusalems (vgl. Apg $2,8-11 ; 6,1.5)$ rekrutierte sich nicht zuletzt auch der Stephanuskreis. ${ }^{55}$ Die Mausoleumsbenutzer verband mit diesem eine liberalere Haltung gegenüber der Tora. Indem sie das Bilderverbot der Tora ignorierten, dazu sich nicht scheuten, den heiligen Gottesnamen auf einen kultisch unreinen Gegenstand in einer Grabesgruft zu schreiben, nahmen sie sich Freiheiten gegenüber der Tora, insbesondere kultbezogenen Geboten, heraus - wie Stephanus, wenn er den Tempelkult kritisierte (Apg 7,48-50.53; vgl. 6,11.13f: Kult- und Torakritik).

52 Für Jerusalemer Provenienz z.B. Schrage, Korinther, 23. Auch wenn Paulus die Glaubensformel in Damaskus oder Antiochien kennengelernt haben sollte, spräche dies nicht gegen Jerusalemer Herkunft. Saßen die Formelautoren freilich doch außerhalb Jerusalems, machte es trotzdem Sinn, eine besondere Nähe zu den Jerusalemer Mausoleumsbenutzern herauszuarbeiten, sowohl sozialgeschichtlich (griechischsprachige Judenchristen) als auch theologisch (Interesse an Auferstehungssymbolik in Jon 2).

53 S. die Argumente für judenchristliche Autorschaft bei Schrage, Korinther, 23.

54 Die vorpaulinische Formel erklärt sich am besten als nicht aus dem Aramäischen übersetzt, sondern als im Ursprung griechisch komponiert. Die schlagenden Argumente bei SCHRAGE, Korinther, 23.

55 Vgl. Theissen, Hellenisten, 323-343; zur auf Jesu Distanz zum Tempel zurückgehenden Tempelkritik des Stephanus DERS., Tempelprophetie, 161-164. 
(c) Weiterhin initiierten die nach dem Stephanusmartyrium aus Jerusalem geflüchteten „Hellenisten“ ${ }^{\text {c56 }}$ als erste eine vorpaulinische Heidenmission (Apg 11,19-26). ${ }^{57}$ Dazu passt, dass ausgerechnet Jona, an dem die „Hellenisten“ des Mausoleums und der 1Kor 15-Formel Interesse zeigten, in Ninive als der erfolgreiche Heidenprophet der Schrift gewirkt hatte, so dass er später in der christlichen Rahmung von 4Esr gar zum Prophet der Heidenkirche stilisiert wurde (s.o.). In gleicher Weise besannen sich die nachösterlichen Trägerkreise des Logions Q 11,31f beim Thema „Heidenumkehr“ ausdrücklich auf Jona, der auf diese Weise gleich in zweierlei Hinsicht - Auferstehung (Q 11,30 s.o.; Ossuar) und Heidenpredigt (Q 11,32) - für frühe nachösterliche Jesusanhänger interessant wurde.

Auch wenn Stephanus mit seinem Jerusalemer Kreis noch nicht Heiden missionierte, scheint er - zum Jona-Heidenprophet-Motiv passend - um den Einschluss der Heiden ins Gottesreich besorgt gewesen zu sein und für die baldige Parusie Jesu ein Öffnen des Tempels für die Heiden erhofft zu haben: ${ }^{58}$ Die Anklage gegen Stephanus wird darauf abgehoben haben, dass er sich gegen die Heiligkeit des Tempels geäußert hatte, um deretwegen Heiden unter Todesandrohung das Betreten des inneren Tempelbezirks verboten war. ${ }^{59}$ Auch vormarkinische Tradition (der „Hellenisten“?) nährte solches Hoffen auf das Öffnen des Tempels für Heiden (Mk 11,17 auf der Basis von Jes 56,7$){ }^{60}$

Ferner behauptet Apg 7,55f, dass Stephanus eine Jesus-MenschensohnChristologie gepflegt habe, die wiederum zum nachösterlichen Verständnis des „Menschensohns“ von Q 11,30 (s.o.) passte.

56 „Hellenisten“ hier wie im lukanischen Sprachgebrauch (Apg 6,1): hellenistische, griechisch-sprachige Judenchristen.

57 Vgl. auch Philippus' Mission in Städten mit paganem Gepräge (Apg 8,5-40): In Sebaste/Samaria wurden sowohl Augustus- als auch Isis-Serapis-Kult gepflegt (z.B. FoERsTER, Architecture, 988f). Die hellenistisch-römische Hafenstadt Caesarea, in der Juden nur ein Quartier im Nordwesten bewohnten, beherbergte mehrere Tempel, darunter einen Augustustempel, sowie zwei Theater und ein Hippodrom (Josephus, Bell I 408-415; Ant XV 331-341; z.B. Foerster, Architecture, 986ff).

58 So Theissen, Tempelprophetie, 161f.168.170f.

$59 \mathrm{Zu}$ den Verbotstafeln OGIS 598 = KüCHLER, Jerusalem, Abb. 175; 378; Josephus, Bell VI 125f; vgl. V 194.

60 Ebenso kann Röm 11,25 dahingehend interpretiert werden, dass der von der „Hellenisten“-Gründung Antiochien geprägte Paulus ersehnte, der innere Tempelbezirk werde noch vor der Parusie den Heiden, insbesondere den Heidenchristen, geöffnet werden. THEISSEN, Tempelprophetie, 168.171: Paulus habe - abweichend von Stephanus - wohl gehofft, dass vor der Parusie der Tempel für die „Fülle der Heiden“, d.h. nicht nur die Proselyten, zugänglich gemacht werden würde. Theißen vergleicht ferner Röm 15,16, wo kultische Terminologie die Motive Heidenchristentum und Tempelkult zumindest metaphorisch aufeinander bezieht. Die Anklage gegen Paulus in Apg 21,29, wonach Paulus den Heidenchristen Trophimus in den inneren Temenosbezirk mitgenommen hätte, wäre dann nicht aus der Luft gegriffen gewesen. 
Nach allem stellen sich deshalb auch die nachösterlichen palästinischen Träger der beiden Jona-Texte Q 11,29f; 11,31f als vierter Personenkreis in die Nähe der drei genannten judenchristlichen Gruppen, das heißt, der griechischsprachigen Mausoleumsleute, der Autoren der griechischen Formel von 1Kor 15 und der „Hellenisten“ im Gefolge des Stephanus. Aber wie nah oder weit war diese Trägergruppe zweier Jona-Logien von den drei anderen Kreisen entfernt? Die beiden Jona-Logien Q 11,29f; 11,31f gehörten kaum zur Grundschicht von $\mathrm{Q},{ }^{61}$ ohne dass dadurch freilich eine Aussage über ihr Alter gemacht wäre (s.o. 1. Teil). Gut möglich ist, dass judenchristliche „Hellenisten“ den vorösterlichen Kernbestand der beiden Jona-Sprüche ins Griechische übersetzten und, wie im 1. Teil gesehen, erweiterten, bevor die griechisch schreibenden (End)redaktoren von Q sie in ihr Dokument aufnahmen.

Unter dem Strich bleibt eine beachtliche Nähe von vier Personenkreisen, die sich partiell überschnitten haben werden. Wenn sichereres Material zur Verfügung stünde, könnte sich zudem die Vermutung verdichten, dass der Agabos des Mausoleums mit dem zeitgleichen christlichen Jerusalemer Propheten Agabos identisch war, der dem Stephanuskreis, insbesondere Philippus und seinen ebenfalls prophezeienden Töchtern, nahestand und gegenüber dem Heidenapostel Paulus, sich um dessen Schicksal sorgend, positiv eingestellt war (Apg 21,8-11). Er hielt Kontakt nicht nur mit Philippus in Caesarea, sondern auch mit der von „Hellenisten“ gegründeten Christengemeinde in Antiochien (Apg 11,27) - alles Indizien, die rechtfertigen könnten, auch ihn dem aufgezeigten Milieu der vier Personenkreise zuzurechnen.

\subsection{Der Fisch als Symbol für den Tod, aus dem Gott errettet}

Im 1. Jh. wurde das Fischsymbol im Umkreis Jerusalems auch auf andere Ossuarien geritzt. Ein Mann namens Clodius (bezeichnenderweise wieder ein griechischer Graffito) fand in einem dieser Gebeinekästchen seine Ruhe (CJO Nr. 348). War er ein Christ? Ein anderer primitiver Fischgraffito (Abb.2), von Figueras ${ }^{62}$ auf einem Ossuar im Lagerbestand der israelischen Antikenbehörde entdeckt, umschließt den hebräisch geschriebenen Namen „Jeshua“ - möglicherweise als Credo des Verstorbenen, der hoffte, wie Jesus

61 Sato, Prophetie, 33-46, rechnet die Verse der letzten Redaktionsstufe zu; Kloppenborg Verbin einer zweiten Redaktionsschicht mit sekundären Erweiterungen (KlopPenBorg Verbin, Excavating, 143-153).

62 Figueras, Ossuaries; CIIP Nr. 546. Das dortige Photo ist schlechter ausgeleuchtet als die unten abgebildete Abb. 2. 
auferweckt zu werden. Dass es sich bei der Kritzelei tatsächlich um einen Fisch handelt, wurde zwar bestritten, ${ }^{63}$ doch zeigt Abb. 2, dass der Schöpfer des Graffitos sich die Mühe machte, ein Maul und einen Schwanz anzudeuten - Elemente, die über ein bloß ornamentales Einrahmen des Jesusnamens hinausgehen. Angesichts des neuen Jona-Fisch-Fundes ist wahrscheinlicher geworden, ${ }^{64}$ dass auch dieser Jeshua-Graffito die Aussage ,Jesus war im Fisch (= Tod) wie Jona und wurde wie dieser daraus errettet" symbolisiert. Alternativ könnte der auf Auferweckung hoffende Verstorbene selbst ein Mann namens Jeshua gewesen sein. ${ }^{65}$

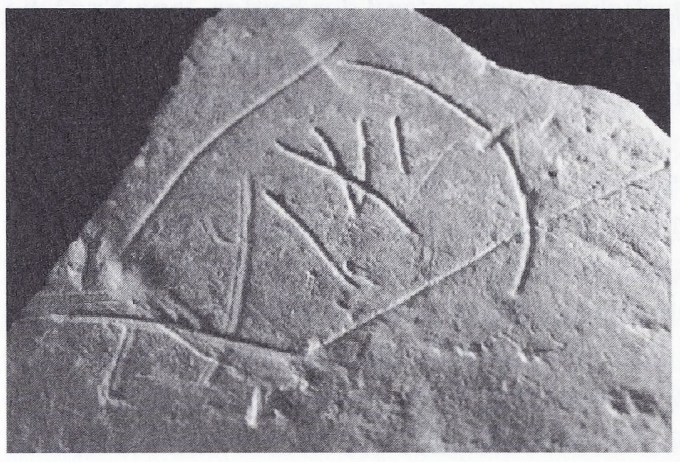

Abb. 2: Jeshua-Graffito (Abdruck mit freundlicher Erlaubnis von James D. Tabor, University of North Carolina/Charlotte, USA).

Im nicht-funeralen Bereich und ohne religiösen Bezug - vielmehr wohl als Hinweis auf die Jerusalemer Essgewohnheiten, wie sie aus den Jerusalemer Latrinenfunden zu erschließen sind - wurden Fische schlussendlich auch im jüdischen Zuhause sozial gehobener Bewohner abgebildet, wie der Fisch auf einem Tischrand im jüdischen Quartier zeigt. ${ }^{66}$

Ornamental und zudem spät (3./4. Jh. n. Chr.) sind die Fischdarstellungen auf acht Bleisärgen in Israel. ${ }^{67}$ Etliche davon, Delphindarstellungen, zeigen deutlich dionysischen Einfluss. ${ }^{68}$ Der Schwarm von vier Fischen als Teil eines Pflanzenornaments ${ }^{69}$ ähnelt dem eines Steinsarkophags von Bet Shearim. ${ }^{70}$ Ebenfalls keine für uns relevanten Belege bieten die unveröffentlichte Diss. von Kancel, Ossuaires, sowie Rahmani, Sarcophagi; DERS., Ossilegium.

Der Fisch als christliches Symbol für den Tod, aus dem Gott wie in der Jonageschichte errettet, ist nicht zu verwechseln mit dem seit dem letzten

63 Vgl. z.B. CJO Nr. 140.

64 So auch TABOR, Report, 25.

$65 \mathrm{Ob}$ auch andere von TABOR, Report, 25f erwogene Belege eines auf Ossuarien umkreisten Namens so zu deuten sind, kann hier dahingestellt bleiben. Die Einkreisung von „Mariamene Mara“ im ca. $45 \mathrm{~m}$ benachbarten „Jesus-Familiengrab" von Talpiyot ähnelt weitaus weniger einem Fisch als die in Abb. 2.

66 Dazu Küchler, Jerusalem, Abb. 305.a.

67 Rahman, Lead Coffins, Nr. 10-12.17f.85f.96; Fig. 48.66B.105f.110f.183.

68 EBD., v.a. Nr. 10.18.86; vgl. z.B. Hesiod, Homer. hymn. 7.

69 EBD., Nr. 96.

70 EBD., S. 39. 
Viertel des zweiten Jahrhunderts beliebten Akrostichon IX $\Theta Y \Sigma$ („Fisch“), das als IH $\Sigma$ OY $\Sigma$ XPI $\Sigma$ TO $\Sigma$ OEOY YIO $\Sigma \Sigma \Omega$ THP den Glauben an Jesus als Gottessohn und Erlöser ausdrückte. ${ }^{71}$ Verschwunden war beim Akrostichon der Bezug auf das Jonabuch. Parallel jedoch existierte auch das Jona-FischMotiv im archäologischen funeralen Kontext weiter (greifbar wieder ab dem 3. Jh.; s.o. Anm. 49), so dass aus zwei verschiedenen Richtungen heraus der Fisch zum prominenten Symbol für die Christen wurde.

\section{Zusammenfassung}

3.1 Die literarische Tradition (Q 11,31f; Q 11,16.29f; 1Kor 15,4; Mt $12,40)$

Das synoptische Jonamotiv entwickelte sich auf mehreren Traditionsstufen, deren erste der historische Jesus legte. Bei Jesus von Nazaret stand Jonas Gerichtspredigt und deren glücklicher Ausgang durch die Umkehr der Niniviter im Zentrum des Interesses (Q 11,31f). Eine Auferstehungssymbolik der Seemonstergeschichte blieb außerhalb des Horizonts. Dieser Fokus entsprach dem Desinteresse des zeitgenössischen Judentums an einer an Jon 2 orientierten Auferstehungssymbolik.

Erste literarische Hinweise auf eine auferstehungstypologische Deutung von Jon 2 bieten die vorpaulinische Formel von 1 Kor 15 aus den dreißiger oder vierziger Jahren sowie Matthäus (Mt 12,40) in seiner Ergänzung von Q. Aber auch für Q 11,30 selbst ist ein solches Verständnis wahrscheinlich, das heißt, plausibler als alternative Interpretationen der Stelle.

Die Überlieferungsgeschichte von Q 11,16.29f wird im Letzten nicht zu klären sein. Es gilt bei diesem Text, viable Rezeptionsmöglichkeiten auf verschiedenen Traditionsstufen - und das heißt, in verschiedenen Kontextuierungen - abzutasten, wobei das Wahrscheinlichkeitsgefälle in folgende Richtung deutet: Während Q 11,16.29a-c, aber genauso gut Q 11,16.29a-d auf der Ebene des historischen Jesus verstehbar sind, wurde Q 11,16.29f erst nach Ostern kommuniziert.

71 Zu diesem Sib. VIII $217 f f$ (Ende des 2. Jh.); Tertullian, bapt. 1, sowie LAMPE, Rome, 29. EBD., V die Abbildung eines entsprechenden IX $\Theta Y \Sigma$-Graffitos vom Ende des 2. Jh. mit einem T-Kreuz zwischen den ersten beiden Buchstaben. 


\subsection{Der Fisch-Jona-Graffito}

Der Graffito auf dem Ossuar wurde wie auch ein zweites, beschriftetes Ossuar aus derselben Felsengruft von

(A) griechischsprachigen Judenchristen Jerusalems geschaffen, die Nähe zu den

(B) Autoren der vorpaulinischen Formel in 1Kor 15, zum

(C) Stephanuskreis und zu den

(D) nachösterlichen Tradenten der beiden Jona-Logien aufwiesen. Beide Logien wurden erst relativ spät in Q eingetragen; die hier interessierenden Träger von Q 11,29f; 11,31f sind deshalb nicht selbstredend mit den Q-Leuten identisch.

Alle vier Personengruppen lebten vor $70 \mathrm{n}$. Chr. und damit vor Mt 12,40. Alle vier verband hellenistisch-judenchristliche Provenienz; mindestens zwei (A.C), wenn nicht alle, Jerusalemer Lokalisation; nachweislich drei (A.B.D) verknüpfte darüber hinaus Interesse an der Jonagestalt. Mit der 1Kor 15-Formel und höchst wahrscheinlich auch mit Q 11,30 verband die Felsengrableute das Interesse an auferstehungstypologischer Deutung von Jon 2 (A.B.D). Stephanuskreis wie auch Grableute (A.C) lebten eine freiheitliche Haltung gegenüber vor allem kultisch orientierten Torageboten (Schreiben des Tetragramms auf einem kultisch unreinen Grabgegenstand, Ignorieren des Bilderverbots). Den Stephanuskreis seinerseits verband mit den Tradenten von Q 11,31f (C.D) das Motiv der Inklusion von Heiden in göttliches Heilshandeln (bei Stephanus Hoffnung auf eschatologische Öffnung des inneren Tempelbezirks für die Heiden; bei seinen Nachfolgern Heidenmission; in Q 11,31f vorbildliche bekehrte Heiden). An Jona interessierte in diesen Kreisen somit nicht nur die auferstehungstypologisch deutbare Seeungeheuergeschichte, sondern auch Jonas erfolgreiche Heidenpredigt. 


\begin{tabular}{|c|c|c|c|c|}
\hline Kriterien & \multicolumn{4}{|c|}{ Gruppen } \\
\hline vor 70 & A & B & C & D \\
\hline $\begin{array}{c}\text { hellenistische Juden- } \\
\text { christen }\end{array}$ & A & B & C & D \\
\hline Jerusalem & A & (B) & C & (D) \\
\hline $\begin{array}{c}\text { Interesse an } \\
\text { Jonagestalt }\end{array}$ & A & B & & D \\
\hline $\begin{array}{c}\text { Interesse an Jon 2- } \\
\text { Auferstehungstypo- } \\
\text { logie }\end{array}$ & A & B & & D \\
\hline $\begin{array}{c}\text { Liberale } \\
\text { Toraauslegung in } \\
\text { Bezug auf Kult }\end{array}$ & A & & C & \\
\hline Inkludierte Heiden & & & C & D \\
\hline
\end{tabular}

\begin{tabular}{|c|l|llll|}
\hline \multicolumn{5}{|c|}{ Kombinationsfrequenzen } \\
\hline $4(-5) \mathrm{mal}$ & A & B & & \\
& A & & & D \\
& & B & D \\
\hline $4 \mathrm{mal}$ & A & & C & \\
\hline $3(-4) \mathrm{mal}$ & & & C & D \\
\hline $2(-3) \mathrm{mal}$ & & B & C & \\
\hline
\end{tabular}

Tab. 2: Übersicht über Querverbindungen zwischen vier Personenkreisen.

Die vier Kreise wenigstens partiell übereinanderzulegen, legt sich nahe. Tab. 2 zeigt die Affinitäten im Überblick. Zumindest darf von einem gemeinsamen "social environment" (,Milieu“) $)^{72}$ der Personenkreise geredet werden. Schlussendlich, nicht mehr als eine Hypothese muss ohne weiteres Fundmaterial bleiben, dass die Lettern АГВ unter dem weissagenden Vierzeiler in der Felsengruft mit dem Jerusalemer Propheten Agabos der Apostelgeschichte zu tun haben, der nach der Emigration der Stephanusnachfolger wiederholten Kontakt zu eben diesen pflegte; er passte gut in das umrissene Milieu.

72 Im Sinne von BARnetT/CASPER, Definition, 465, die u.a. definieren: „Human social environments encompass the immediate physical surroundings, social relationships, and cultural milieus within which defined groups of people function and interact." 


\section{Literatur}

Avni, G./Greenhut, Z./Ilan, T., Three New Burial Caves of the Second Temple Period in Aceldama (Kidron Valley), in: H. Geva (Hg.), Ancient Jerusalem Revealed, Jerusalem ${ }^{2} 2000,206-218$.

BARNETT, E./CASPER, M., A Definition of „Social Environment“", American Journal of Public Health 91/3 (2001), 465.

BaUCKham, R., On the Patio Tomb Inscriptions, in: http:/www.patheos.com/ blogs/bibleandculture/2012/04/17/richard-bauckham-on-the-patio-tomb-inscriptions/.

BAYER, H.E., Jesus' Predictions of Vindication and Resurrection. The Provenance, Meaning and Correlation of the Synoptic Predictions (WUNT II/20), Tübingen 1986.

BeCKer, J., Jesus von Nazaret, Berlin 1996.

Cotton, H.M. u.a. (Hg.), Corpus Inscriptionum Iudaeae/Palaestinae. Bd. I/1, Berlin $2010[=$ CIIP $]$.

Dormeyer, D., Das Neue Testament im Rahmen der antiken Literaturgeschichte.

Eine Einführung, Darmstadt 1993.

Figueras, P., Decorated Jewish Ossuaries (DMOA 20), Leiden 1983.

Foerster, G., Art and Architecture in Palestine, in: S. Safrai/M. Stern (Hg.), The Jewish People in the First Century. Bd. 2, Assen/Amsterdam 1976, 971-1006. Goodenough, E.R., Jewish Symbols in the Greco-Roman Period. Bd. 2 (BollS

37), New York 1965.

Hoffmann, P./Heil, C. (Hg.), Die Spruchquelle Q, Darmstadt/Leuven 2002.

JoRDAN, D. R., Notes from Carthage, ZPE 111 (1996), 115-123.

KAnCEL, D., Les ossuaires juifs au tournant de notre ère, Bd. I-II, Hist.-Philolog.

Diss., Paris (ohne Datum).

Kloner, A., Survey of Jerusalem. The Southern Sector, Jerusalem 2000.

-/Zissu, B., The Necropolis of Jerusalem in the Second Temple Period (Interdisciplinary Studies in Ancient Culture and Religion 8), Leuven/Dudley 2007.

Kloppenborg, J., The Formation of Q. Trajectories in Ancient Wisdom Collections, Philadelphia 1987.

Kloppenborg Verbin, J. S., Excavating Q. The History and Setting of the Sayings Gospel, Minneapolis 2000.

Koch, K., Messias und Menschensohn. Die zweistufige Messianologie der jüngeren Apokalyptik, JBTh 8 (1993), 73-102.

KüCHLER, M., Jerusalem. Ein Handbuch und Studienreiseführer zur Heiligen Stadt (OLB IV/2), Göttingen 2006.

LAMPE, P., MEXPI THC CHMEPON. A New Edition of Matthew 27:64b; 28:13 in Today's Pop Science and a Salty Breeze from the Dead Sea, in: Ders./M. Mayordomo/M. Sato (Hg.), Neutestamentliche Exegese im Dialog. 
Hermeneutik - Wirkungsgeschichte - Matthäusevangelium (FS U. Luz), Neukirchen-Vluyn 2008, 355-366.

-, From Paul to Valentinus. Christians at Rome in the First Two Centuries, London/Minneapolis ${ }^{4} 2010$.

Lehmann, K., Auferweckt am dritten Tag nach der Schrift. Früheste Christologie,

Bekenntnisbildung und Schriftauslegung im Lichte von 1 Kor. 15,3-5 (QD

38), Freiburg i.Br. ${ }^{2} 1969$.

LÜHRMANN, D., Die Redaktion der Logienquelle (WMANT 33), NeukirchenVluyn 1969.

LuZ, U., Das Evangelium nach Matthäus. Bd. 2: Mt 8-17 (EKK I/2), Zürich/

Neukirchen-Vluyn 1990.

Mora, V., Le signe de Jonas, Paris 1983.

Nestori, A., Repertorio Topografico delle Pitture delle Catacombe Romane (RSCr 5), Vatikanstadt 1975.

RaHMANI, L.Y., A Catalogue of Jewish Ossuaries in the Collections of the State of Israel, Jerusalem 1994 [= CJO].

-, A Catalogue of Roman and Byzantine Lead Coffins from Israel, Jerusalem 1999. -, Ossuaries and Ossilegium (Bone-Gathering) in the Late Second Temple Period, in: H. Geva (Hg.), Ancient Jerusalem Revealed, Jerusalem ${ }^{2} 2000,191-205$.

-, Sarcophagi of the Late Second Temple Period in Secondary Use, in: H. Geva

(Hg.), Ancient Jerusalem Revealed, Jerusalem 22000, 231-234.

SAFraI, S., Home and Family, in: Ders./M. Stern (Hg.), The Jewish People in the

First Century. Bd. 2, Assen/Amsterdam 1976, 728-792.

SAto, M., Q und Prophetie. Studien zur Gattungs- und Traditionsgeschichte der Quelle Q (WUNT II/29), Tübingen 1988.

SchmitT, G., Das Zeichen des Jona, ZNW 69 (1978), 123-129.

Schrage, W., Der Erste Brief des Paulus an die Korinther. Bd. 4: 1Kor 15,116,24 (EKK VII/4), Neukirchen-Vluyn/Zürich 2001.

Schürmann, H., Gottes Reich - Jesu Geschick. Jesu ureigener Tod im Licht seiner Basileia-Verkündigung, Freiburg i.Br. 1983.

Snyder, G.F., Ante Pacem. Archaeological Evidence of Church Life Before Constantine, Macon 2003.

TABOR, J.D., A Preliminary Report of a Robotic Camera Exploration of a Sealed 1st Century Tomb in East Talpiot, Jerusalem, Feb. 2012, updated version March 16, 2012, online: http://www.bibleinterp.com/PDFs/Jonah_2012.pdf.

-, The Hebrew Name „YONAH“ Embedded in the Image of the Fish on the

Talpiot Tomb Ossuary, April 11, 2012, online: http://www.bibleinterp.com/ PDFs/Jonah_2012.pdf.

Theissen, G., Hellenisten und Hebräer (Apg 6,1-6). Gab es eine Spaltung der Urgemeinde?, in: H. Lichtenberger (Hg.), Geschichte - Tradition - Reflexion.

Bd. 3: Frühes Christentum (FS M. Hengel), Tübingen 1996, 323-343.

-, Die Bedeutung der Tempelprophetie Jesu für die ersten Christen. Die

Wirkungsgeschichte der Tempelprophetie im 1. Jh. n.Chr., in: Ders. u.a. 
(Hg.), Jerusalem und die Länder. Ikonographie - Topographie - Theologie (FS M. Küchler) (NTOA 70), Göttingen 2009, 149-201.

VögtLe, A., Die „Gretchenfrage“ des Menschensohnproblems. Bilanz und Perspektive (QD 152), Freiburg i.Br. 1994. 